\title{
Cerebral autosomal dominant arteriopathy with subcortical infarcts and leukoencephalopathy (CADASIL) during pregnancy: case report and review of the literature
}

\begin{abstract}
Background: Cerebral autosomal dominant arteriopathy with subcortical infarcts leukoencephalopathy (CADASIL) is a hereditary disease characterized by early adult-onset of neurologic manifestations such as migraine, dementia, and stroke. The prevalence of CADASIL is 4.15 per 100 000adults. Pregnancy and puerperium are associated with worsening of symptoms.

Case: We present a 31-year-old G3P0020 patient with a pre-pregnancy diagnosis of CADASIL followed during pregnancy and postpartum period. The patient had a strong family history of CADASIL as well as neurologic symptoms of headaches and vision changes. The patient was started on aspirin and her pregnancy was managed by a multidisciplinary team. She was admitted at 34 weeks for an evaluation of severe headaches and lower limb weakness. She had an uncomplicated cesarean delivery at 39 weeks and an uncomplicated postpartum period.

Discussion: We report a case of a pregnant woman with CADASIL and review the literature published on this topic. We discuss the management of CADASIL during pregnancy and postpartum, as well as the perioperative management in patients with this genetic disease.
\end{abstract}

Volume 2 Issue I - 2017

\author{
Mauricio Francisco La Rosa', Oxford \\ Corrina, ${ }^{2}$ Sehdev Harish ${ }^{2}$ \\ 'Department of Obstetrics and Gynecology, University of Texas \\ Medical Branch, USA \\ 2Department of Obstetrics and Gynecology, Pennsylvania \\ Hospital, USA
}

Correspondence: Mauricio Francisco La Rosa, Department of Obstetrics and Gynecology, University of Texas Medical Branch, 3I I I Avenue Q. Galveston, 77550, USA, Tel 215-518-0694, Email mauricio.la.rosa@gmail.com

Received: February 14, 2017| Published: March 15, 2017
Abbreviations: CADASIL, cerebral autosomal dominant arteriopathy with subcortical infarcts Leukoencephalopathy, CVA, cerebrovascular accidents

\section{Introduction}

Cerebral autosomal dominant arteriopathy with subcortical infarcts and leukoencephalopathy (CADASIL) is a disease caused by a mutation on NOTCH3 gene. Initially discovered in the 1990's, the estimated prevalence of CADASIL in the United Kingdom is 4.15 per 100000 adults. ${ }^{1}$ CADASIL has become an important cause of ischemic cerebrovascular accidents (CVA) in young patients. This pathology has been associated with $2 \%$ of lacunar stroke with leucoaraiosis in patients younger than 65 and $11 \%$ in patients younger than 50 years old. ${ }^{2}$

The NOTCH3 gene encodes information for NOTCH3 receptors production in the vascular smooth muscle cells. There are almost 700 types of mutations of this gene but only few have been reported to cause CADASIL. These mutations cause arteriopathy of small vessels, usually of cerebral penetrating arteries, characterized by recurrent subcortical lacunar infarcts and white matter degeneration. This disease is also associated with gray matter micro hemorrhages and laminar cortical neuronal apoptosis. ${ }^{3}$ Histological findings include non-hypertensive, non-atherosclerosis, and non-amyloid small caliber vasculopathy. The presence of granular osmiophilic material in the tunica media is considered pathognomonic.

There is a wide range of severity of the disease phenotypes, and no genotype-phenotype relationship. Variability on phenotypic expression causes misdiagnosis and under diagnosis. A report of monochorionic twins with CADASIL suggested that lifestyle and environmental factors can change the clinical manifestations of the same mutation by epigenetic alterations such as DNA methylation and histone modification. ${ }^{4}$

The most common clinical manifestation is CVA, present in 60$80 \%$ of all cases. The average age for the first CVA is 49 years old. Hypertension, smoking, and high blood viscosity have been proposed as three major risk factors for stroke in CADASIL patients, described in more than half of the cases. One third of these patients suffer with migraines, commonly preceded by severe, long-lasting aura. This is five times more common than general population. ${ }^{1}$

A review of MRI studies from patients with CADASIL showed that all patients, including the asymptomatic group, had radiologic alterations. They suggested that different subcortical areas had different vulnerabilities to ischemia in CADASIL and that age effect may show an accumulation of lesions with aging during the course of the disease

There is scant data on the impact of pregnancy in patients with CADASIL. We present a case a patient with CADASIL during pregnancy and review the literature.

\section{Case}

A 31-year-old G3P0020 was referred to maternal fetal medicine at 13 weeks of gestational age. She was diagnosed with migraines at age 19. The patient reported several episodes of headaches associated with loss of peripheral vision. When she was 23 years old and after a severe headache, a brain MRI showed multiple patchy areas of signal 
abnormality in the cerebral white matter. At that point, the patient was diagnosed and treated for multiple sclerosis. The patient denied any personal history of CVA or dementia. Her obstetric history was notable for two prior first trimester induced abortions. Family history was significant CVA in her maternal grandfather had at 55years of age. Her mother was found to have a NOTCH3 gene mutation on chromosome 19 during a migraine work up. Per the patient, her mother never had a CVA or symptoms of dementia.

The patient was diagnosed before her third pregnancy and was asymptomatic for five years prior to this pregnancy. She denied taking any medication and reported smoking 2 cigarettes per day. During the MFM consult, the patient was found to have live singleton intrauterine pregnancy. Extensive genetic counseling was performed but the patient refused all types of fetal genetic screening for CADASIL. Aspirin $81 \mathrm{mg}$ daily was started and the patient was extensively counseled to stop smoking.

A follow up ultrasound at 20 weeks of gestation showed no structural evidence of fetal malformation. Serial growth ultrasounds remained within normal limits.

At 34weeks of gestational age the patient was admitted to the hospital due to severe right eye pressure-like headache associated with transient right upper limb weakness for 24 hours. Her blood pressures were normal and preeclampsia was ruled out. The physical exam performed by the neurologist was significant for mild flattening of right nasolabial fold. There were no other abnormal neurologic or psychiatric findings. The MRI (Figure 1) showed multiple small foci of abnormal signal in the cerebral white matter consistent with micro vascular ischemic changes but no acute pathology. This episode was self-limited and the patient was discharged home in stable condition after 10days.

Her prenatal course was otherwise uncomplicated and she had an elective low transverse cesarean section with bilateral tubal ligation at 39 weeks, under spinal anesthesia. There were no complications during the procedure and the postpartum period.
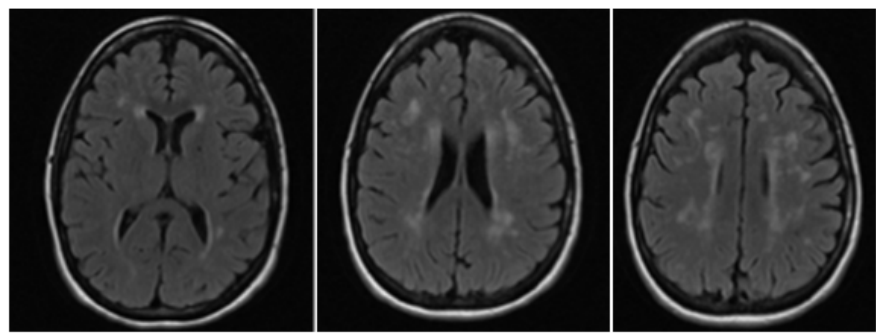

Figure I MRI performed at 34.0weeks of gestation.

\section{Comments}

Pregnant women have several physiologic, biochemical and anatomic changes that can impact the clinical manifestations of CADASIL. Pregnancy and postpartum are hypercoagulable states, known to increase the levels of von Will brand, factor VIII, fibrinogen, and plasminogen activator inhibitors.

In 2005, the incidence of stroke during pregnancy and puerperium in the US was 34.2per 100000 deliveries, threefold higher than in 15 to 44 -year-old non-pregnant women. Preeclampsia and eclampsia were related to $45 \%$ of that cases.
CADASIL is an important cause of ischemic CVA in young populations. The main manifestations of this disorder include transient cerebral ischemic attack, decreased cognitive status, and vascular dementia. In a retrospective case series of 25 women with CADASIL and history of prior pregnancies, $80 \%$ of complications included neurologic symptoms and preeclampsia, mostly during postpartum. The mean ages of complicated and uncomplicated pregnancies were 31.6 and 24.7years, respectively. Hemiparesthesia, aphasia, hemiparesis, and visual disorders were also common. Preeclampsia was more prevalent in CADASIL patients $(10 \%)$ than in normal pregnancies $(3-5 \%)$. There was no difference in miscarriage rate, gestational age at delivery, or mode of delivery. ${ }^{6}$

The most common lesions seen on MRI occur in the per ventricular and deep white matter. Lesions are initially nodular or punctiform and become diffuse and symmetric. The most suggestive areas affected by CADASIL are the external capsule and anterior temporal lobe. As opposed to multiple sclerosis, basal ganglia and thalamus are also affected. The severity is higher in symptomatic patients and increases dramatically with age.

Currently, there are no recommendations about prenatal care or management for people with CADASIL. There is no specific treatment for this disease but some experts recommend ant platelet drugs, as well as acetazolamide for migraines. ${ }^{7}$ In cases of stroke, thrombolytic agents must be used with caution since they can increase the risk of preterm labor, placental abruption, fetal death, and postpartum hemorrhage. Adib-Samii et al. ${ }^{8}$ suggested these patients should receive antihypertensive treatment if needed and lifestyle changes should be encouraged (e.g., stop smoking) prior to conception. ${ }^{8}$

Regarding the perioperative management, there is insufficient information available to recommend an anesthetic management in these patients. Currently, CADASIL is not considered a contraindication for regional anesthesia. ${ }^{7,9}$ Hypovolemia should be avoided with adequate fluid infusion. In case of general anesthesia, it is crucial to maintain normocapnia. Postpartum pain control can be achieved with NSAIDs as well as opioids. ${ }^{9}$ we suggest that patients with CADASIL be monitored closely during pregnancy, in a high risk obstetric service, providing a multidisciplinary care including neurologists and anesthesiologists.

\section{Acknowledgements}

None.

\section{Conflict of interest}

Author declares that there is no conflict of interest.

\section{References}

1. Chabriat H, Joutel A, Dichgans M, et al. Cadasil. Lancet Neurol. 2009;8(7):643-653.

2. Dichgans M, Mayer M, Uttner I, et al. The phenotypic spectrum of CADASIL: clinical findings in 102 cases. Ann Neurol. 1998;44(5):731-739.

3. Chabriat H, Levy C, Taillia H, et al. Patterns of MRI lesions in CADASIL. Neurology. 1998;51(2):452-457.

4. Mykkänen K, Junna M, Amberla K, et al. Different clinical phenotypes in monozygotic CADASIL twins with a novel NOTCH3 mutation. Stroke. 2009;40(6):2215-2218. 
5. James AH, Bushnell CD, Jamison MG, et al. Incidence and risk factors for stroke in pregnancy and the puerperium. Obstet Gynecol. 2005;106(3):509-516.

6. Roine S, Pöyhönen M, Timonen S, et al. Neurologic symptoms are common during gestation and puerperium in CADASIL. Neurology. 2005;64(8):1441-1143.

7. Errando CL, Navarro L, Vila M, Pallardó MA. Anesthetic management of a patient diagnosed with CADASIL (cerebral arteriopathy, autosomal dominant, with subcortical infarcts and leukoencephalopathy). Rev Esp Anestesiol Reanim. 2012;59(2):102-106.
8. Adib Samii P, Brice G, Martin RJ, et al. Clinical spectrum of CADASIL and the effect of cardiovascular risk factors on phenotype: study in 200 consecutively recruited individuals. Stroke. 2010;41(4):630-634.

9. Dieu JH, Veyckemans F. Perioperative management of a CADASIL type arteriopathy patient. Br J Anaesth. 2003;91(3):442-444. 